\title{
Synthesis and cytotoxic evaluation of malachite green derived oleanolic and ursolic acid piperazineamides
}

\author{
Sander Friedrich ${ }^{1} \cdot$ Immo Serbian $^{1} \cdot$ Sophie Hoenke ${ }^{1} \cdot$ Ratna Kancana Wolfram $^{1} \cdot$ René Csuk $^{1}$
}

Received: 23 January 2020 / Accepted: 24 March 2020 / Published online: 9 April 2020

(c) The Author(s) 2020

\section{Abstract}

The coupling of acetylated piperazinylamide spacered triterpenoic oleanolic acid and ursolic acid with meta or para substituted carboxylated malachite green analogs gave conjugates 10, 11, 15, and $\mathbf{1 6}$ that were cytotoxic for several human tumor cell lines. Especially, an oleanolic acid-derived compound $\mathbf{1 0}$ was cytotoxic for MCF-7 human breast carcinoma cells $\left(\mathrm{EC}_{50}=0.7 \mu \mathrm{M}\right)$. These derivatives represent first examples of triterpenoic acid derivatives holding a cationic scaffold derived from malachite green.

\section{Graphical Abstract}

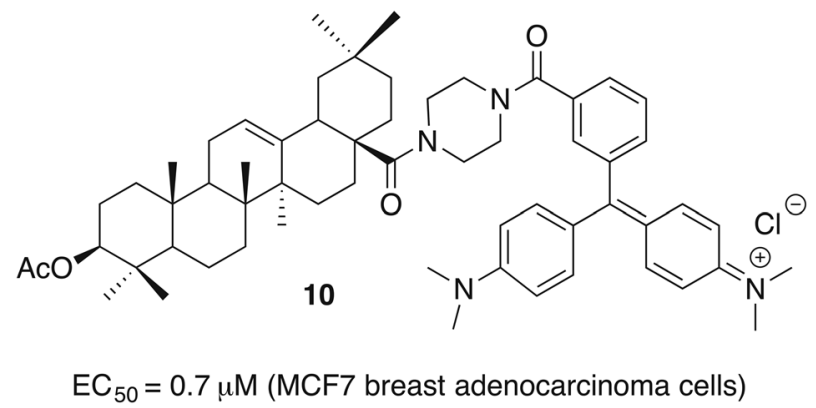

Keywords Oleanolic acid $\cdot$ Ursolic acid $\cdot$ Cytotoxicity $\cdot$ Terpenoids $\cdot$ Malachite green

\section{Introduction}

Chemotherapeutic treatment of cancer represents still a scientific challenge. Although nowadays many patients suffering from cancer can be cured or-at least-their life span can be increased. Several types of cancer, however, are

Supplementary information The online version of this article (https:// doi.org/10.1007/s00044-020-02536-1) contains supplementary material, which is available to authorized users.

René Csuk

rene.csuk@chemie.uni-halle.de

1 Organic Chemistry, Martin-Luther-University Halle-Wittenberg, Kurt-Mothes-Str. 2, D-06120 Halle (Saale), Germany difficult to be handled, and the cure rate (Holzel et al. 2017; Laffman-Johnson 2012; Vliek et al. 2018) remained low over all the years despite intensive research.

Recently the potential of scaffolds holding a cationic functional group came into the focus of renewed interest inasmuch as their transport into cells might be facilitated by cation-transporters (Cai et al. 2016; Everett et al. 2013; Qian et al. 2016). Some of these transporters were reported to be overexpressed in malignant cells (Cai et al. 2014; Cai et al. 2016; Everett et al. 2013). Several molecules holding a cationic residue are cytotoxic and seem to target the mitochondria, such as ammonium (Biedermann et al. 2010; Kataev et al. 2014) or phosphonium salts (Spivak et al. 2013, 2017) of more complex molecules. Thus, these compounds are mitocans ("mitochondrially targeted anticancer drugs"). 
Some conjugates of pentacyclic triterpenes are mitocans, too. Thus, hybrids holding an extra cationic functional group have shown promising to excellent cytotoxic results. While "simple" quaternary ammonium salts (Biedermann et al. 2010; Kataev, Strobykina, and Zakharova 2014) were only of moderate cytotoxicity with their $\mathrm{EC}_{50}$ values being in the same potency range as phosphonium salts (Spivak et al. 2017, 2013), hybrids consisting of a suitable pentacyclic triterpene, an amine spacer and a BODIPY-FL group (Brandes et al. 2020; Krajcovicova et al. 2018) held lower $\mathrm{EC}_{50}$ values against a variety of different human cancer cell lines. Superior cytotoxicity, however, was found for those triterpenoids holding one or two $O$-acetyl groups on ring A, an amide spacer at $\mathrm{C}-28$ (preferentially a piperazinyl residue), and a rhodamine B moiety attached to this spacer (Sommerwerk et al. 2017). EC 50 values in the low micromolar (Kahnt et al. 2018; Wolfram et al. 2018a, 2018b) and even nano-molar range (Sommerwerk et al. 2017) were reported for these conjugates.

\section{Results and discussion}

In extension of these findings and due to the close structural similarity between malachite green (A) and rhodamine B (B, Fig. 1) we became interested in the synthesis and biological evaluation of conjugates holding a cationic triphenylmethane moiety especially of scaffolds of the "malachite green type", i.e., $\mathbf{1}$ and $\mathbf{2}$; these scaffolds differ from malachite green by the presence of an additional carboxyl group; the latter is necessary for the attachment to the triterpene-spacer adduct.

Recently conjugates holding this type of a malachite green moiety (Müller et al. 1997; Rassow and Gruber 1915; Yang et al. 2007) have been used for the production of antibodies to be used in environmental analyses (Yang et al. 2007), as part of a fluorophore to label antimicrobial peptides (Zhao et al. 2016) and for the detection of nerve gas simulants by chromogenic chemodosimeters (Costero et al. 2012).

The synthesis of $\mathbf{1}$ (Scheme 1) started with the reaction of $m$-formyl-benzoic acid (3) with $N, N$-dimethylaniline in the presence of zinc chloride to yield 4 (Rassow and Gruber 1915; Sinev et al. 1978). Similarly, from $p$-formylbenzoic acid (5) compound $\mathbf{6}$ was obtained in $89 \%$ yield as a blue-greenish compound. Reaction of $\mathbf{4}$ with tetrachloro- $p$-benzoquinone afforded $41 \%$ of 1 while from 6 (Harle et al. 2018; Mueller et al. 1981; Rassow and Gruber 1915) under the same conditions 2 was obtained; both compounds are dark green solids. Compounds of this type are also known as "Mordant Green" or "Mordant Blue".
Fig. 1 Structure of malachite green (a) and rhodamine B (b) as well as of scaffolds $\mathbf{1}$ and $\mathbf{2}$. The lower section displays the close structural similarity between malachite green and rhodamine B<smiles>CN(C)c1ccc(C(=C2C=CC(=[N+](C)Cl)C=C2)c2ccccc2)cc1</smiles>

A<smiles>CN(C)c1ccc(C(=C2C=CC(=[N+](C)Cl)C=C2)c2cccc(C(=O)O)c2)cc1</smiles><smiles>CCN(CC)c1ccc2c(-c3ccccc3C(=O)O)c3ccc(=[N+](C)CC)cc-3oc2c1</smiles><smiles></smiles><smiles>CN(C)c1ccc(C(=C2C=CC(=[N+](C)Cl)C=C2)c2ccc(C(=O)O)cc2)cc1</smiles>

2

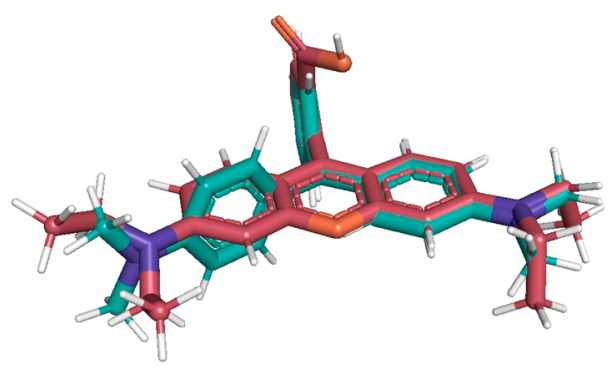


Scheme 1 Synthesis of malachite green analogs $\mathbf{1}$ and $\mathbf{2}$ : reactions and conditions: a $\mathrm{ZnCl}_{2}, \mathrm{EtOH}, N, N-$ dimethylaniline, reflux, 1 day, $89 \%$ (of 4), 69\% (of 6); b tetrachloro- $p$-benzoquinone, $\mathrm{CHCl}_{3}, 35^{\circ} \mathrm{C}, 2 \mathrm{~h}, 41 \%$ (of $\mathbf{1}$ ), $65 \%$ (of 2)

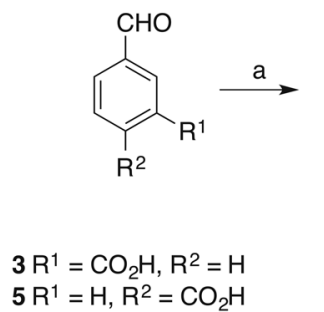

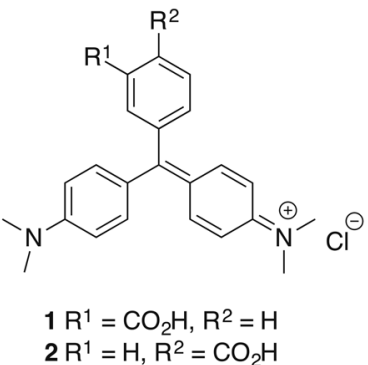

Oleanolic acid (7) was acetylated (Sommerwerk et al. 2017) (Scheme 2) to yield acetate 8; the latter compound was coupled with piperazine as previously reported to yield 9 (Sommerwerk et al. 2017). Compound 1 was activated in situ with oxalyl chloride and coupled with 9 to afford target compound 10. Reaction of 9 with 2 gave 11.

Acetylation of ursolic acid (12) gave acetate 13 followed by its reaction with piperazine to yield amide 14 (Sommerwerk et al. 2017). Coupling of the latter compound with 1 or 2 as described above afforded the hybrids $\mathbf{1 5}$ and 16, respectively.

The compounds were screened for their cytotoxic activity using sulforhodamine B assays; the results from these assays are summarized in Table 1. Betulinic acid and doxorubicin were used as standards. While ursolic acid (12) is of similar cytotoxicity as standard betulinic acid, no significant cytotoxicity was found for oleanolic acid (7). Cytotoxicity increased about tenfold for the actylated piperazinylamides 9 and 14. Except for the HT29 human adenocarcinoma cells, the $\mathrm{EC}_{50}$ values determined for the carboxylated malachite green derivatives $\mathbf{1}$ and $\mathbf{2}$ were found in the low one-digit $\mu \mathrm{M}$ range. As far as the coupling products 10, 11, 15, and 16 are concerned, oleanolic acid-derived compounds $\mathbf{1 0}$ and $\mathbf{1 1}$ were of significantly higher cytotoxicity than ursolic acidderived 15 and 16. The selectivity between tumor cells and the nonmalignant fibroblasts is - by and large-the same as in betulinic acid but significantly better than that of doxorubicin. Furthermore, meta substituted malachite green carboxylates 10 and 15 were about twice as cytotoxic than para substituted analogs $\mathbf{1 1}$ and 16, respectively. Thus, this makes oleanolic acid-derived $\mathbf{1 0}$ to the most cytotoxic compound of this series holding $\mathrm{EC}_{50}$ values between 0.7 and $0.9 \mu \mathrm{M}$. Furthermore, 10 is $\sim 17$ times more cytotoxic than standard triterpenoic acid betulinic acid and circa 50 times more cytotoxic than starting material oleanolic acid. Interestingly, while the lowest cytotoxicity was observed for HT29 cells, for an analogous rhodamine B derivative especially for this cell line the highest cytotoxicity has been noted. This seems to prove that both the type of triterpenoic acid, the type of amide linkage, the type of cationic residue, and its substitution pattern are of great importance both with respect to a tumor cell line-specific cytotoxicity and to cytotoxicity in general. Ongoing studies in our laboratory try to gain a deeper insight into these observations.

\section{Conclusion}

Coupling of acetylated triterpenoic oleanolic acid and ursolic acid holding a piperazinylamide spacer with meta or para substituted carboxylated malachite green analogs gave conjugates 10, 11, 15, and 16, respectively. These compounds were cytotoxic for several human tumor cell lines. Thereby, oleanolic acid-derived compound $\mathbf{1 0}$ was especially cytotoxic for MCF-7 human breast carcinoma cells $\left(\mathrm{EC}_{50}=0.7 \mu \mathrm{M}\right)$.

\section{Experimental}

NMR spectra were recorded using the Varian spectrometers Gemini 2000 or Unity 500 ( $\delta$ given in ppm, $J$ in Hz; typical experiments: H-H-COSY, HMBC, HSQC, NOESY), MS spectra were taken on a Finnigan MAT LCQ 7000 (electrospray, voltage $4.1 \mathrm{kV}$, sheath gas nitrogen) instrument. The optical rotations were measured on a Perkin-Elmer polarimeter at $20^{\circ} \mathrm{C}$; TLC was performed on silica gel (Merck 5554, detection with cerium molybdate reagent); melting points are uncorrected (Leica hot stage microscope), and elemental analyses were performed on a FossHeraeus Vario EL (C-HNS) unit. IR spectra were recorded on a Perkin Elmer FT-IR spectrometer Spectrum 1000. The solvents were dried according to usual procedures. The purity of the compounds was determined by HPLC and found to be $>96 \%$. Oleanolic acid (7) and ursolic acid (12) were obtained from Betulinines (Stř́brná Skalice, Czech Republic) in bulk quantities.

\section{Synthesis}

\section{$\mathrm{N}$-(4-((3-carboxyphenyl)(4-(dimethylamino)phenyl) methylene)cyclohexa-2,5-dien-1-yliden)- $N$ - methylmethanaminium chloride (1)}

Following the procedure given for the synthesis of $\mathbf{2}$ from 4 (1.34 mmol, $500 \mathrm{mg}$ ), tetrachloro- $p$-benzoquinone $(393 \mathrm{mg}, 1.6 \mathrm{mmol})$ and glacial acetic acid $(1 \mathrm{~mL}$; $0.1 \mathrm{mmol}$ ) followed by crystallization of the solid from water (100 mL) 1 (224 mg, 41\%) was obtained as a purple 
Scheme 2 Synthesis of compounds 7-16: reactions and conditions: a $\mathrm{Ac}_{2} \mathrm{O}, \mathrm{NEt}_{3}$, DMAP, DCM, $88 \%$ (of 8 ), $64 \%$ (of 13); b oxalyl chloride, THF; DCM, piperazine $49 \%$; c oxalyl chloride, THF; $\mathrm{NEt}_{3}$, DMAP, $25^{\circ} \mathrm{C}, 2 \mathrm{~h}, 50 \%$; d oxalyl chloride, THF; $\mathrm{NEt}_{3}$, DMAP, $25^{\circ} \mathrm{C}, 2 \mathrm{~h}, 50 \%$; e oxalyl chloride, THF; DCM, piperazine $57 \%$; f oxalyl chloride, THF; $\mathrm{NEt}_{3}$, DMAP, $25^{\circ} \mathrm{C}, 2 \mathrm{~h}, 50 \%$; g oxalyl chloride, THF; $\mathrm{NEt}_{3}$, DMAP, $25^{\circ} \mathrm{C}, 2 \mathrm{~h}, 41 \%$

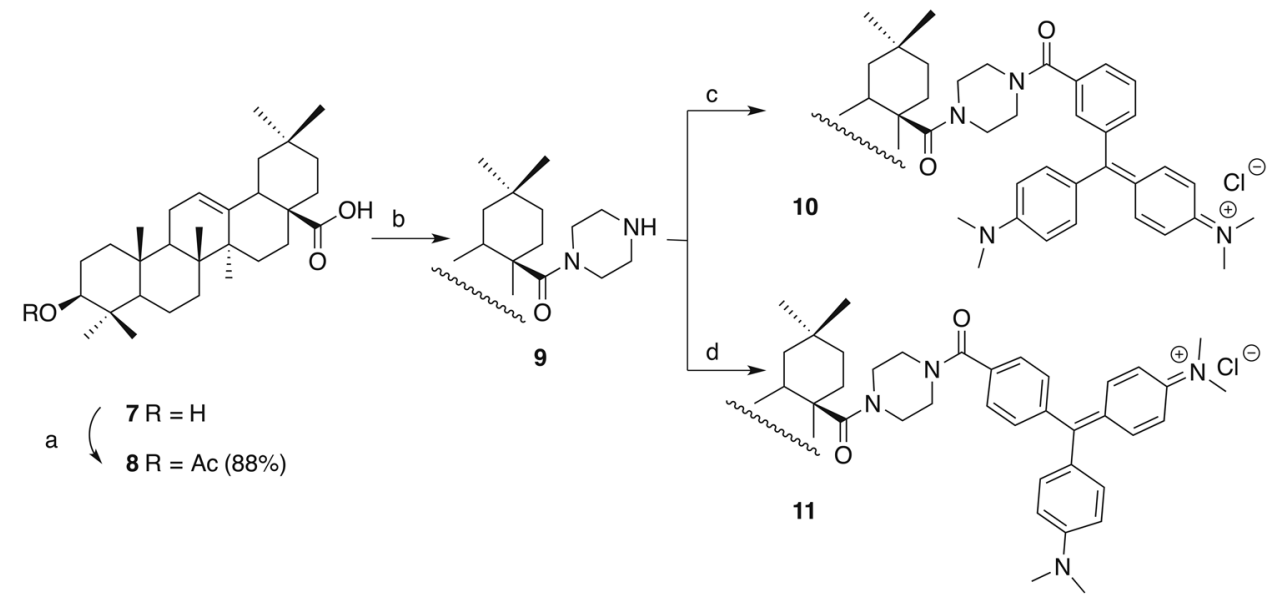

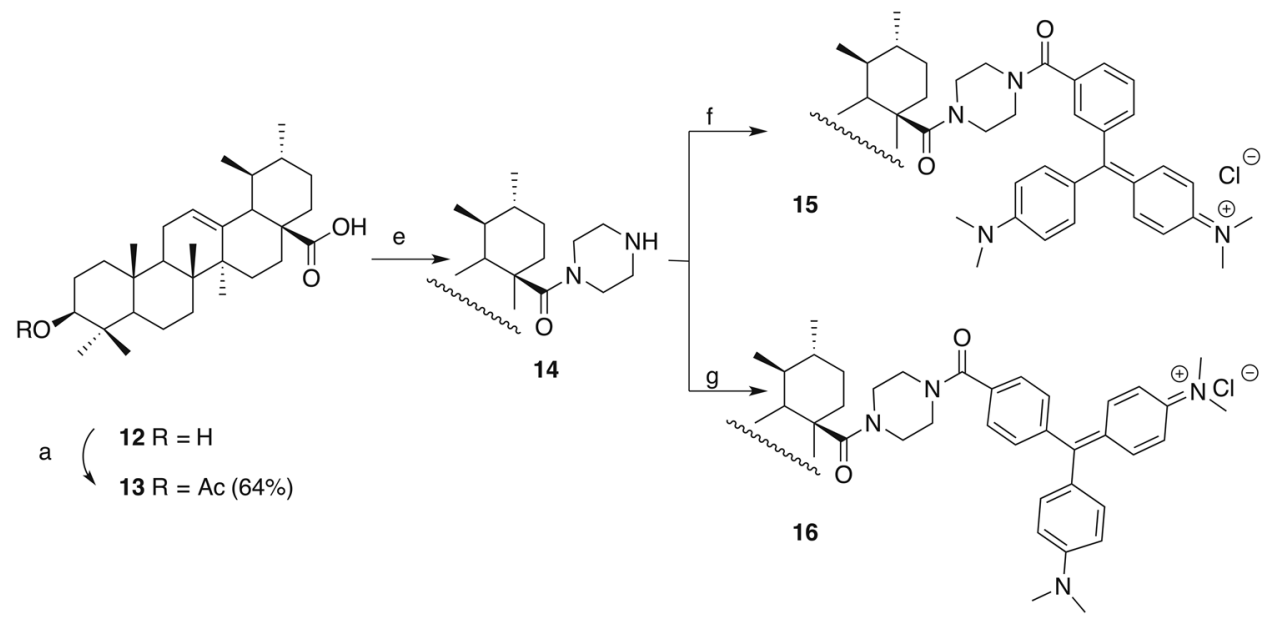

Table 1 Cytotoxicity of selected compounds; SRB assay $\mathrm{EC}_{50}$ values $[\mu \mathrm{M}]$ after $72 \mathrm{~h}$ of treatment; averaged from three independent experiments performed each in triplicate; confidence interval $\mathrm{CI}=95 \%$

\begin{tabular}{lllllll}
\hline$\#$ & FaDu & A2780 & HT29 & MCF-7 & SW1736 & NIH 3T3 \\
\hline $\mathbf{1}$ & $6.1 \pm 1.0$ & $9.7 \pm 1.1$ & $18.2 \pm 1.4$ & $6.4 \pm 0.8$ & $8.1 \pm 1.5$ & $6.3 \pm 1.7$ \\
$\mathbf{2}$ & $8.2 \pm 0.9$ & $4.6 \pm 0.7$ & $12.4 \pm 1.2$ & $6.5 \pm 1.1$ & $9.7 \pm 0.8$ & $5.2 \pm 1.1$ \\
$\mathbf{7}$ & $>30$ & $>30$ & $>30$ & $>30$ & $>30$ & $>30$ \\
$\mathbf{9}$ & n.d. & $1.7 \pm 0.1$ & $1.3 \pm 0.1$ & $1.7 \pm 0.1$ & $1.8 \pm 0.9$ & $2.2 \pm 1.0$ \\
$\mathbf{1 0}$ & $0.8 \pm 0.2$ & $0.9 \pm 0.2$ & $4.3 \pm 0.5$ & $0.7 \pm 0.1$ & $0.9 \pm 0.1$ & $0.6 \pm 0.2$ \\
$\mathbf{1 1}$ & $1.5 \pm 0.1$ & $2.1 \pm 0.3$ & $4.6 \pm 0.2$ & $1.4 \pm 0.3$ & $1.7 \pm 0.1$ & $1.5 \pm 0.3$ \\
$\mathbf{1 2}$ & n.d. & $11.7 \pm 0.6$ & $10.6 \pm 0.7$ & $12.7 \pm 0.1$ & $14.3 \pm 0.4$ & $5.2 \pm 1.4$ \\
$\mathbf{1 4}$ & n.d. & $2.1 \pm 0.1$ & $1.9 \pm 0.3$ & $2.0 \pm 0.1$ & $3.1 \pm 0.8$ & $9.7 \pm 0.8$ \\
$\mathbf{1 5}$ & $1.5 \pm 0.1$ & $1.4 \pm 0.3$ & $2.4 \pm 0.2$ & $1.2 \pm 0.1$ & $1.7 \pm 0.4$ & $1.2 \pm 0.3$ \\
$\mathbf{1 6}$ & $2.3 \pm 0.2$ & $4.9 \pm 0.6$ & $7.9 \pm 0.5$ & $2.9 \pm 0.7$ & $4.0 \pm 0.5$ & $2.6 \pm 0.7$ \\
Betulinic acid & n.d. & $12.7 \pm 1.8$ & $18.4 \pm 2.0$ & $12.0 \pm 1.7$ & $16.4 \pm 1.9$ & $16.1 \pm 1.4$ \\
Doxorubicin & n.d. & $0.01 \pm 0.01$ & $0.9 \pm 0.2$ & $1.1 \pm 0.3$ & $1.7 \pm 0.3$ & $0.008 \pm 0.001$ \\
\hline
\end{tabular}

Human cancer cell lines: FaDu (hypopharyngeal carcinoma), A2780 (ovarian carcinoma), HT29 (colorectal carcinoma), MCF-7 (breast adenocarcinoma), SW1736 (thyroid carcinoma) NIH 3T3 (nonmalignant fibroblasts); cutoff $30 \mu \mathrm{M}$

n.d. not determined 
solid; $R_{F}=\left(\mathrm{ACN} / \mathrm{H}_{2} \mathrm{O}, 85: 15\right)=0.44 ;$ m.p. $207-210{ }^{\circ} \mathrm{C}$; ${ }^{1} \mathrm{H}$ NMR $\left(400 \mathrm{MHz}, \quad\right.$ DMSO-d $\left.{ }_{6}\right): \delta=10.23(\mathrm{~s}, \quad 1 \mathrm{H}$, $\mathrm{COOH}), 8.25\left(\mathrm{~d}, J=7.8 \mathrm{~Hz}, 1 \mathrm{H}, 4^{\prime}-\mathrm{H}\right), 7.80(\mathrm{t}, J=$ $\left.1.8 \mathrm{~Hz}, 1 \mathrm{H}, 2^{\prime}-\mathrm{H}\right), 7.73\left(\mathrm{t}, J=7.7 \mathrm{~Hz}, 1 \mathrm{H}, 5^{\prime}-\mathrm{H}\right), 7.56(\mathrm{dt}$, 7.7, $\left.1.5 \mathrm{~Hz}, 1 \mathrm{H}, 6^{\prime}-\mathrm{H}\right), 7.34-7.29$ (m, 4H, 2-H, 7-H), 7.08 $(\mathrm{d}, J=9.3 \mathrm{~Hz}, 4 \mathrm{H}, 3-\mathrm{H}, 8-\mathrm{H}) \mathrm{ppm} ;{ }^{13} \mathrm{C}$ NMR $(400 \mathrm{MHz}$, DMSO-d $\left.\mathrm{d}_{6}\right): \quad \delta=174.0 \quad(\mathrm{C}-5), 172.4 \quad(\mathrm{C}-\mathrm{OOH}), 167.0$ (C-9), 156.9 (C-1), 140.5 (C-7), 138.5 (C-6'), 134.9 (C$\left.2^{\prime}\right), 133.5\left(\mathrm{C}-4^{\prime}\right), 131.6\left(\mathrm{C}-1^{\prime}\right), 129.6\left(\mathrm{C}-3^{\prime}\right), 126.9(\mathrm{C}-6$, C4), $121.1\left(\mathrm{C}-5^{\prime}\right), 114.7$ (C-3, C8), $41.0\left(1-\mathrm{NMe}_{2}\right), 21.5$ $\left(9-\mathrm{NMe}_{2}\right) \mathrm{ppm}$; IR (KBr): $\nu=3447 \mathrm{sbr}, 1617 w, 1584 m$, $1369 m, 1168 \mathrm{~cm}^{-1}$; UV-Vis $(\mathrm{CHCl} 3): \lambda_{\max }(\log \varepsilon)=$ 220 (3.4), 345.17 (2.82), 475.22 (2.72), 682.72 (3.06), $714.92(3.27) \mathrm{nm}$; MS (ESI, MeOH): $m / z(\%)=373.33$ (100\%, [M-Cl] $]^{-}$); analysis calcd for $\mathrm{C}_{24} \mathrm{H}_{25} \mathrm{~N}_{2} \mathrm{O}_{2} \mathrm{Cl}$ (408.93): C 70.49, H 6.16, N 6.85; found: C 70.35, H $6.38, \mathrm{~N} 6.73$.

\section{$\mathrm{N}$-(4-((4-carboxyphenyl)(4-(dimethylamino)phenyl) methylene)cyclohexa-2,5-dien-1-yliden)- $\mathrm{N}$ - methylmethanaminium chloride (2)}

To a solution of $6(500 \mathrm{mg} 1.34 \mathrm{mmol})$ and tetrachloro-pbenzoquinone $(393 \mathrm{mg}, 1.6 \mathrm{mmol})$ in $\mathrm{CHCl}_{3}(50 \mathrm{~mL})$ glacial acetic acid $(1 \mathrm{~mL}, 0.1 \mathrm{mmol})$ was added, and stirring at $35^{\circ} \mathrm{C}$ was continued for $2 \mathrm{~h}$. The volatiles were removed under reduced pressure, and the residue was crystallized from water $(100 \mathrm{~mL})$ to yield $2(970 \mathrm{mg}, 65 \%)$ as a dark green solid; $R_{F}=0.3\left(\mathrm{ACN} / \mathrm{H}_{2} \mathrm{O}, 85: 15\right)$; m.p. $230-232{ }^{\circ} \mathrm{C}$; ${ }^{1} \mathrm{H}$ NMR $\left(400 \mathrm{MHz}, \mathrm{DMSO}-\mathrm{d}_{6}\right): \delta=10.21(\mathrm{~s}, 1 \mathrm{H}, \mathrm{COOH})$, $8.12\left(\mathrm{~d}, J=8.7 \mathrm{~Hz}, 2 \mathrm{H}, 3^{\prime}-\mathrm{H}\right), 7.42\left(\mathrm{~d}, J=8.5 \mathrm{~Hz}, 2 \mathrm{H}, 2^{\prime}-\right.$ $\mathrm{H}), 7.32(\mathrm{~d}, J=9.2 \mathrm{~Hz}, 2 \mathrm{H}, 3-\mathrm{H}), 7.08(\mathrm{~d}, J=9.2 \mathrm{~Hz}, 2 \mathrm{H}$, 2-H), 6.97 (d, $J=9.2 \mathrm{~Hz}, 2 \mathrm{H}, 7-\mathrm{H}), 6.61(\mathrm{~d}, J=9.2 \mathrm{~Hz}, 2 \mathrm{H}$, 8-H), 3.31 (s, 12H, 1-NMe 2 ), 2.85 (s, 6H, 9-NMe 2 ) ppm; ${ }^{13} \mathrm{C}$ NMR $\left(100 \mathrm{MHz}\right.$, DMSO-d $\left.{ }_{6}\right): \delta=173.6(\mathrm{C}-5), 166.7$ (C-OOH), 156.7 (C-1), 154.3 (C-9), 140.3 (C-3), 136.0 (C1'), 134.9 (C-2'), 134.3 (C-4'), $129.7\left(\mathrm{C}-3^{\prime}\right), 128.9$ (C-7), 121.0 (C-6), 111.9 (C-8), $41.1\left(1-\mathrm{NMe}_{2}\right), 40.6\left(9-\mathrm{NMe}_{2}\right)$ ppm; IR (KBr): $\nu=3447 \mathrm{~s}, 1589 \mathrm{~m}, 1363 \mathrm{~m}, 1169 \mathrm{~m} \mathrm{~cm}^{-1}$; UV-vis (CHCl3): $\lambda_{\max }(\log \varepsilon)=345$ (4.38), 480 (4.14), 675 (4.43), 725 (4.65) nm; MS (ESI, MeOH): $\mathrm{m} / z(\%)=373.33$ (100, $\left.[\mathrm{M}-\mathrm{Cl}]^{-}\right)$; analysis calcd for $\mathrm{C}_{24} \mathrm{H}_{25} \mathrm{~N}_{2} \mathrm{O}_{2} \mathrm{Cl}$ (408.93): C 70.49, H 6.16, N 6.85; found: C 70.30, H 6.32, N 6.69 .

\section{3-[Bis(4-dimethylamino)phenyl]methyl-benzoic acid (4)}

To a solution of $3(1 \mathrm{~g}, 6.67 \mathrm{mmol})$ and $\mathrm{ZnCl}_{2}(2.7 \mathrm{~g}$, $20.01 \mathrm{mmol})$ in ethanol $(50 \mathrm{~mL}) \mathrm{N}, \mathrm{N}$-dimethylaniline $(2.5 \mathrm{~g}, 20.01 \mathrm{mmol})$ was added, and stirring under reflux was continued for 1 day. Work-up as described for $\mathbf{6}$ gave $\mathbf{4}$ $(2.23 \mathrm{~g}, 89 \%)$ as a greenish/blue solid; $R_{F}=0.89$ ( $n$-hexane/

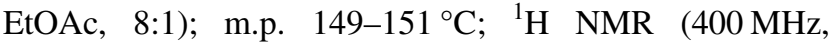

DMSO-d $\left.{ }_{6}\right): \delta=7.92\left(\mathrm{~d}, J=13.8 \mathrm{~Hz}, 2 \mathrm{H}, 2^{\prime}-\mathrm{H}, 4^{\prime}-\mathrm{H}\right), 7.29$ (d, $\left.J=19.0 \mathrm{~Hz}, 2 \mathrm{H}, 6^{\prime}-\mathrm{H}, 3^{\prime}-\mathrm{H}\right), 6.94(\mathrm{~d}, J=13.8 \mathrm{~Hz}, 4 \mathrm{H}$, 3-H), $6.67(\mathrm{~d}, J=13.8 \mathrm{~Hz}, 4 \mathrm{H}, 2-\mathrm{H}), 5.4(\mathrm{~s}, 1 \mathrm{H}, 5-\mathrm{H}), 2.88$ (s, $12 \mathrm{H}, 1-\mathrm{NMe}_{2}$ ) ppm; ${ }^{13} \mathrm{C}-\mathrm{NMR}$ (400 MHz, DMSO-d ${ }_{6}$ ): $\delta=148.8(\mathrm{C}-1), 145.5\left(\mathrm{C}-1^{\prime}\right), 134.1\left(\mathrm{C}-2^{\prime}\right), 132.8\left(\mathrm{C}-3^{\prime}\right)$, $131.1\left(\mathrm{C}-5^{\prime}\right), 129.9$ (C-3, C-4), 129.1 (C-6'), $128.1\left(\mathrm{C}-4^{\prime}\right)$, 113.0 (C-2), 54.9 (C-5), $40.9\left(\mathrm{NMe}_{2}\right)$ ppm; IR (KBr): $\nu=$ $3448 s, 1614 m, 1519 m, 1351 w \mathrm{~cm}^{-1}$; UV-vis $\left(\mathrm{CHCl}_{3}\right): \lambda_{\max }$ $(\log \varepsilon)=289.65(4.3 \mathrm{~nm})$; MS (ESI, MeOH): $m / z(\%)=$ $375.27\left(100 \%,[\mathrm{M}+\mathrm{H}]^{+}\right)$; analysis calcd for $\mathrm{C}_{24} \mathrm{H}_{24} \mathrm{~N}_{2} \mathrm{O}_{2}$ (374.48): C 76.98, H 7.00, N 7.48; found: C 76.83, H 7.15, N 7.31.

\section{4-[Bis(4-dimethylamino)phenyl]methyl-benzoic acid (6)}

To a solution of $5(1.44 \mathrm{~g}, 9.6 \mathrm{mmol})$ and $\mathrm{ZnCl}_{2}(3.9 \mathrm{~g}$, $28.8 \mathrm{mmol})$ in ethanol $(100 \mathrm{~mL}) \mathrm{N}, \mathrm{N}$-dimethylaniline (3.6 $\mathrm{mL}, 28.8 \mathrm{mmol}$ ) was slowly added, and the mixture was stirred under reflux for 1 day. The mixture was diluted with methanol $(40 \mathrm{~mL})$, the $\mathrm{pH}$ value was adjusted to 5 by adding aq. $\mathrm{HCl}$, and the precipitate was filtered off. Compound $6(2.45 \mathrm{~g}, 69 \%)$ was obtained as light green/blue solid; $R_{F}=0.95 \quad(n$-hexane/EtOAc, $3: 1) ; \quad$ m.p. $252{ }^{\circ} \mathrm{C}$ (decomp.) (lit: $250{ }^{\circ} \mathrm{C}$, decomp. (Yang et al. 2007)); ${ }^{1} \mathrm{H}$ NMR $\left(400 \mathrm{MHz}\right.$, DMSO-d 6 ): $\delta=12.76\left(\mathrm{~s}, 1 \mathrm{H}, 5^{\prime}-\mathrm{H}\right)$, 7.88-7.82 (m, 2H, 3'-H), 7.21-7.16 (m, 2H, 2'-H), 6.93-6.85 (m, 4H, 2-H), 5.38 (s, 1H, 5-H), 2.83 (s, 12H, $\left.\mathrm{NMe}_{2}\right)$ ppm; ${ }^{13} \mathrm{C}$ NMR $(400 \mathrm{MHz}$, DMSO-d 6 ): $\delta=167.8$ $\left(\mathrm{C}-5^{\prime}\right), 151.1\left(\mathrm{C}-1^{\prime}\right), 149.3$ (C-1), 131.9 (C-4), 129.8 (C-2), $129.7\left(\mathrm{C}-3^{\prime}\right), 129.4\left(\mathrm{C}-2^{\prime}\right), 112.8\left(\mathrm{C}-3^{\prime}\right), 54.59$ (C-5), 40.7 $\left(\mathrm{NMe}_{2}\right) \mathrm{ppm}$; IR (KBr): $\nu=3447 s_{b r}, 2883 m, 1683 s, 1610 s$, $1571 w, 1519 s, 1479 w, 1443 w, 1428 w, 1349 w, 1314 w$, $1293 w, 1227 w, 1202 w, 1177 w, 1164 w, 1061 w, 1017 w \mathrm{~cm}^{-1}$; UV-vis $\left(\mathrm{CHCl}_{3}\right): \lambda_{\max }(\log \varepsilon)=270.37$ (4.16) nm; MS (ESI, $\mathrm{MeOH}): m / z(\%)=375.33\left(100 \%,[\mathrm{M}+\mathrm{H}]^{+}\right)$; anlysis calcd for $\mathrm{C}_{24} \mathrm{H}_{24} \mathrm{~N}_{2} \mathrm{O}_{2}$ (374.48): C 76.98, $\mathrm{H}$ 7.00, N 7.48; found: C 76.77, H 7.19, N 7.29.

\section{3ß-Acetyloxy-olean-12-en-28-oic acid (8)}

Acetylation of $\mathbf{7}$ as previously described gave $\mathbf{8}(88 \%)$ as a colorless solid; $R_{F}=0.7$ (silica gel, toluene/ethyl acetate/formic acid/heptane, 80:26:5:1); m.p.: $263-265^{\circ} \mathrm{C}$ (lit.: 260-261 ${ }^{\circ} \mathrm{C}$ ) (Sommerwerk et al. 2017); $[\alpha]_{\mathrm{D}}=$ $+117.7^{\circ}$ (c 0.37, $\left.\mathrm{CHCl}_{3}\right)$, (lit.: $+119^{\circ}$ (c 0.1, $\mathrm{CHCl}_{3}$ ) (Sommerwerk et al. 2017); MS (ESI, MeOH): $m / z=497.5$ $\left(75 \%,[\mathrm{M}-\mathrm{H}]^{-}\right), 995.2\left(100 \%, \quad[2 \mathrm{M}-\mathrm{H}]^{-}\right), 1017.7$ $\left(29 \%,[2 \mathrm{M}-2 \mathrm{H}+\mathrm{Na}]^{-}\right)$.

\section{3ß-Acetyloxy-olean-12-en-28-oyl piperazine (9)}

Reaction of $\mathbf{8}$ with oxalyl chloride and piperazine as previously described gave 9 (49\%); $R_{F}=0.46$ (silica gel, 
$\left.\mathrm{CHCl}_{3} / \mathrm{MeOH}, 9: 1\right)$; m.p. $172-176{ }^{\circ} \mathrm{C}$ (lit.: $170-176^{\circ} \mathrm{C}$ ) (Sommerwerk et al. 2017); MS (ESI, MeOH): $m / z=567.4$ $\left(45 \%,[\mathrm{M}+\mathrm{H}]^{+}\right)$.

\section{$\mathrm{N}$-(4-((4-dimethylamino)phenyl)-3-((4-(3ß-acetyloxyolean- 12-en-28-carboxy)piperazin)-1-yl)oxy)- carbonylphenylmethylencyclohexa-2,5-dien-1-yliden)- $\mathrm{N}-$ methylmethanaminium chloride (10)}

Following the procedure given for the synthesis of 11, from 1 (200 mg, $0.49 \mathrm{mmol})$ compound $\mathbf{1 0}$ (240 mg, 49.8\%) was obtained as a dark green solid; $R_{F}\left(\mathrm{CHCl}_{3} / \mathrm{MeOH}, 9: 1\right)=$ 0.35 ; m.p. $212-215{ }^{\circ} \mathrm{C} ;{ }^{1} \mathrm{H}$ NMR $\left(400 \mathrm{MHz}, \mathrm{CDCl}_{3}\right): \delta=$ $8.02(\mathrm{~s}, 1 \mathrm{H}, 37-\mathrm{H}), 7.78-7.69(\mathrm{~m}, 1 \mathrm{H}, 39-\mathrm{H}), 7.49-7.3(\mathrm{~m}$, $\left.4 \mathrm{H}, 2^{\prime}-\mathrm{H}, 7^{\prime}-\mathrm{H}\right), 7.05-6.91(\mathrm{~d}, J=8.9 \mathrm{~Hz}, 1 \mathrm{H}, 40-\mathrm{H}), 6.99$ (d, $\left.J=8.8 \mathrm{~Hz}, 4 \mathrm{H}, 3^{\prime}-\mathrm{H}, 8^{\prime}-\mathrm{H}\right), 6.72-6.58(\mathrm{~m}, 1 \mathrm{H}, 38-\mathrm{H})$, $5.21(\mathrm{t}, J=3.6 \mathrm{~Hz}, 1 \mathrm{H}, 12-\mathrm{H}), 4.49(\mathrm{~m}, 1 \mathrm{H}, 3-\mathrm{H}), 3.72(\mathrm{q}, J$ $=7.0 \mathrm{~Hz}, 4 \mathrm{H}, 33-\mathrm{H}), 3.39\left(\mathrm{~s}, 6 \mathrm{H}, \mathrm{N}-\mathrm{Me}_{2}\right), 3.23(\mathrm{~d}, J=$ $15.5 \mathrm{~Hz}, 4 \mathrm{H}, 34-\mathrm{H}), 2.96(\mathrm{~s}, 1 \mathrm{H}, 18-\mathrm{H}), 2.17(\mathrm{~m}, 1 \mathrm{H}, 16-$ $\left.\mathrm{H}_{\mathrm{a}}\right), 2.04(\mathrm{~s}, 3 \mathrm{H}, 32-\mathrm{H}), 1.94-1.86(\mathrm{~m}, 2 \mathrm{H}, 11-\mathrm{H})$, 1.63-1.54 (m, 8H, 6-H, 16- $\left.\mathrm{H}_{\mathrm{b}}, 2-\mathrm{H}, 15-\mathrm{H}_{\mathrm{a}}, 7-\mathrm{H}\right), 1.53-1.20$ $\left(\mathrm{m}, 11 \mathrm{H}, 22-\mathrm{H}, 15-\mathrm{H}_{\mathrm{b}}, 21-\mathrm{H}, 1-\mathrm{H}, 19-\mathrm{H}, 9-\mathrm{H}\right), 0.79(\mathrm{~m}, 1 \mathrm{H}$, 5-H), 0.89-0.80 (m, 12H, 23-H, 24-H, 25-H, 27-H), 0.72 (s, $3 \mathrm{H}, 30-\mathrm{H}), 0.70(\mathrm{~s}, 6 \mathrm{H}, 26-\mathrm{H}, 29-\mathrm{H}) \mathrm{ppm} ;{ }^{13} \mathrm{C}$ NMR $\left(100 \mathrm{MHz}, \mathrm{CDCl}_{3}\right): \delta=171.0(\mathrm{C}-28), 171.0(\mathrm{C}-31), 169.3$ (C-35), $157.0\left(\mathrm{C}-1^{\prime}, \mathrm{C}^{\prime} 9^{\prime}\right), 143.6\left(\mathrm{C}-7^{\prime}\right), 140.9\left(\mathrm{C}-8^{\prime}\right), 140.8$ (C-2'), 138.5 (C-41), 134.9 (C-39), 133.4 (C-37), 131.5 (C40), 129.5 (C-38), 125.0 (C-12), 121.9 (C-36), 120.4 (C-6'), $114.3\left(\mathrm{C}-3^{\prime}\right), 81.3(\mathrm{C}-3), 56.0$ (C-30), 47.4 (C-9), 46.6 (C34), 43.4 (C-33), $41.2\left(1-\mathrm{NCH}_{3}\right), 39.4$ (C-8), 39.0 (C-5), 38.8 (C-17), 38.6 (C-1), 37.5 (C-4), 36.5 (C-10), 36.0 (C18), 34.9 (C-21), 33.3 (C-22), 31.9 (C-14), 30.4 (C-20), 30.3 (C-27), 28.3 (C-24), 23.7 (C-15), 23.6 (C-2), 23.5 (C11), 23.4 (C-19), 23.0 (C-16), 21.6 (C-32), 21.2 (C-29), 18.4 (C-6), 17.0 (C-23), 16.1 (C-26), 15.9 (C-25), 8.4 (C-7), 0.89 (C-5') ppm; IR (KBr): $\nu=3447 s_{b r}, 2925 m, 1618 m$, $1584 m, 1370 m, 1247 m, 1170 \mathrm{~m} \mathrm{~cm}^{-1}$; UV-vis $(\mathrm{CHCl} 3)$ : $\lambda_{\max }(\log \varepsilon)=229.01$ (3.56), 244.02 (4.26), 347.2 (3.87), 485.3 (3.71), 731.09 (4.35) nm; MS (ESI, MeOH): $\mathrm{m} / z$ (\%): $921.73\left(100 \%,[\mathrm{M}-\mathrm{Cl}]^{-}\right) ; \mathrm{C}_{26} \mathrm{H}_{81} \mathrm{~N}_{4} \mathrm{O}_{4} \mathrm{Cl}$ (957.78): $\mathrm{C}$ 75.24, H 8.52, N 5.85; found: C 75.13, H 8.81, N 5.43.

\section{$N$-(4-((4-dimethylamino)phenyl)-4-((4-(3ß-acetyloxyolean- 12-en-28-carboxy)piperazin)-1-yl)oxy)- carbonylphenylmethylencyclohexa-2,5-dien-1-yliden)- $\mathrm{N}$ - methylmethanaminium chloride (11)}

To a solution of $2(500 \mathrm{mg}, 1.23 \mathrm{mmol})$ in dry dichloromethane (DCM, $50 \mathrm{~mL})$, oxalyl chloride $(0.62 \mathrm{mg}$, $4.92 \mathrm{mmol}$ ) and three drops of dry dimethylformamide (DMF) were added. After stirring for $30 \mathrm{~min}$, the volatiles were removed under reduced pressure, the residue was dissolved in dry tetrahydrofuran (THF, $3 \times 20 \mathrm{~mL}$ ), and the solvents were evaporated DMF. The solid was dissolved in dry DCM $(50 \mathrm{~mL})$, triethylamine $(0.2 \mathrm{~g}, 0.3 \mathrm{~mL}, 10 \mathrm{mmol})$, DMAP (4-dimethylaminopyridine, $15 \mathrm{mg}, 0.1 \mathrm{mmol}$ ), and a solution of $9(849 \mathrm{mg}, 1.5 \mathrm{mmol})$ in dry DCM $(20 \mathrm{~mL})$ were added. The mixture was stirred at $25^{\circ} \mathrm{C}$ for $2 \mathrm{~h}$; usual workup followed by chromatography (silica gel, $\mathrm{CHCl}_{3} / \mathrm{MeOH}$, 9:1) gave $11(594 \mathrm{mg}, 50 \%)$ as a dark green solid; $R_{F}$ $\left(\mathrm{CHCl}_{3} / \mathrm{MeOH}, 9: 1\right)=0.35$; m.p. $192-195{ }^{\circ} \mathrm{C} ;{ }^{1} \mathrm{H}$ NMR $\left(400 \mathrm{MHz}, \mathrm{CDCl}_{3}\right): \delta=7.99(\mathrm{~s}, 2 \mathrm{H}, 37-\mathrm{H}), 7.56(\mathrm{~d}, J=$ $8.2 \mathrm{~Hz}, 2 \mathrm{H}, 38-\mathrm{H}), 7.37$ (dd, $J=8.73,3.5 \mathrm{~Hz}, 4 \mathrm{H}, 2^{\prime}-\mathrm{H}, 8^{\prime}-$ $\mathrm{H}), 6.97\left(\mathrm{~d}, J=9.2 \mathrm{~Hz}, 4 \mathrm{H}, 3^{\prime}-\mathrm{H}, 7^{\prime}-\mathrm{H}\right), 5.21(\mathrm{~m}, 1 \mathrm{H}, 12-$ H), 4.47 (t, $J=8.3 \mathrm{~Hz}, 1 \mathrm{H}, 3-\mathrm{H}), 3.69(\mathrm{~m}, 4 \mathrm{H}, 33-\mathrm{H}), 3.36$ (s, 6H, N- $\mathrm{Me}_{2}$ ), 3.1 (q, $\left.J=14.0,7.3 \mathrm{~Hz}, 4 \mathrm{H}, 34-\mathrm{H}\right), 2.94$ (s, $1 \mathrm{H}, 18-\mathrm{H}), 2.12\left(\mathrm{~m}, 1 \mathrm{H}, 16-\mathrm{H}_{\mathrm{a}}\right), 2.02(\mathrm{~s}, 3 \mathrm{H}, 32-\mathrm{H}), 1.89$ (m, 2H, 11-H), 1.79-1.54 (m, 8H, 6-H, 16- $\mathrm{H}_{\mathrm{b}}, 2-\mathrm{H}, 15-\mathrm{H}_{\mathrm{a}}$, 7-H), 1.53-1.25 (m, 8H, 22-H, 21-H, 1- $\left.\mathrm{H}_{\mathrm{a}}, 19-\mathrm{H}, 9-\mathrm{H}\right)$, $1.22(\mathrm{~s}, 3 \mathrm{H}, 27-\mathrm{H}), 1.06\left(\mathrm{~m}, 2 \mathrm{H}, 1-\mathrm{H}_{\mathrm{b}}, 15-\mathrm{H}_{\mathrm{b}}\right), 0.92(\mathrm{~m}, 6 \mathrm{H}$, 25-H, 29-H), 0.79 (m, 1H, 5-H), 0.88-0.77 (dd, $J=10.9$, $6.2 \mathrm{~Hz}, 9 \mathrm{H}, 23-\mathrm{H}, 24-\mathrm{H}, 30-\mathrm{H}), 0.72$ (s, 3H, 26-H) ppm; ${ }^{13} \mathrm{C}$ NMR $\left(100 \mathrm{MHz}, \mathrm{CDCl}_{3}\right): \delta=171.0(\mathrm{C}-28), 169.3(\mathrm{C}-35)$, 162.5 (C-31), $157.0\left(\mathrm{C}-1^{\prime}, \mathrm{C}^{\prime}\right), 143.6\left(\mathrm{C}-7^{\prime}\right), 140.9\left(\mathrm{C}-8^{\prime}\right)$, $140.8\left(\mathrm{C}-2^{\prime}\right), 140.8$ (C-13), 139.3 (C-39), 134.7 (C-36), 127.3 (C-38), 127.2 (C-37), 125.2 (C-12), $119.4\left(\mathrm{C}-6^{\prime}\right)$, 114.1 (C-3'), 80.9 (C-3), 55.3 (C-30), 47.5 (C-9), 45.9 (C34), 42.1 (C-33), $41.2\left(1-\mathrm{NMe}_{2}\right), 39.4$ (C-8), 38.8 (C-5), 38.7 (C-17), 38.2 (C-1), 37.7 (C-4), 36.9 (C-10), 36.5 (C18), 34.4 (C-21), 33.0 (C-22), 31.4 (C-14), 30.4 (C-20), 29.7 (C-27), 28.1 (C-24), 24.1 (C-2), 23.5 (C-15), 23.3 (C19), 23.0 (C-16), 21.3 (C-32), 21.2 (C-29), 18.2 (C-6), 16.9 (C-23), 16.7 (C-26), 15.5 (C-25), 8.6 (C-7), 0.98 (C-5') ppm; IR (KBr): $\nu=3445 s_{b r}, 2956 w, 1636 m, 1584 m$, $1371 \mathrm{~m}, 1170 w \mathrm{~cm}^{-1}$; UV-vis $(\mathrm{CHCl}): \lambda_{\max }(\log \varepsilon)=242$ (3.16), 345 (3.83), 475 (3.61), 617.15 (1.79), $732.12 \mathrm{~nm}$ (3.22) nm; MS (ESI, MeOH): $m / z(\%)=921.80(100 \%$, [M $-\mathrm{Cl}]^{-}$); analysis calcd for $\mathrm{C}_{26} \mathrm{H}_{81} \mathrm{~N}_{4} \mathrm{O}_{4} \mathrm{Cl}$ (957.78): C 75.24, H 8.52, N 5.85; found: C 75.02, H 8.76, N 5.39.

\section{3ß-Acetyloxy-urs-12-en-28-oic acid (13)}

Acetylation of $\mathbf{1 2}$ as previously described (Sommerwerk et al. 2017) gave $13(64 \%)$ as a colorless solid; $R_{F}=0.7$ (silica gel, toluene/ethyl acetate/formic acid/heptane, 80:26:5:1); m.p. $242-244{ }^{\circ} \mathrm{C}$ (lit.: $242.7-244.1^{\circ} \mathrm{C}$ ); $[\alpha]_{\mathrm{D}}=$ $+69.89^{\circ}\left(c\right.$ 0.86, $\left.\mathrm{CHCl}_{3}\right)$, (lit.: $+71.2^{\circ}\left(c 1.0, \mathrm{CHCl}_{3}\right)$; MS (ESI, MeOH): $m / z=497.5\left(64 \%,[\mathrm{M}-\mathrm{H}]^{-}\right), 542.9(30 \%$, $\left.\left[\mathrm{M}+\mathrm{HCO}_{2}\right]^{-}\right), 995.1\left(68 \%,[2 \mathrm{M}-\mathrm{H}]^{-}\right), 1017.5(100 \%$, $\left.[2 \mathrm{M}-2 \mathrm{H}+\mathrm{Na}]^{-}\right)$.

\section{3ß-Acetyloxy-olean-12-en-28-oyl piperazine (14)}

Reaction of $\mathbf{1 3}$ with oxalyl chloride and piperazine as previously (Sommerwerk et al. 2017) described gave 14 (57\%); $R_{F}=0.44$ (silica gel, $\mathrm{CHCl}_{3} / \mathrm{MeOH}, 9: 1$ ); m.p. $158-161^{\circ} \mathrm{C}$ 
(lit.: $\left.158-161^{\circ} \mathrm{C}\right)$; $\mathrm{MS}$ (ESI, MeOH): $m / z=567.4(61 \%$, $\left.[\mathrm{M}+\mathrm{H}]^{+}\right)$.

$\mathrm{N}$-(4-((4-dimethylamino)phenyl)-3-((4-(3ß-acetyloxyurs-12en-28-carboxy)piperazin1-1-yl)oxy)carbonylphenylmethylencyclohexa-2,5-dien-1-yliden)- $N$ methylmethanaminium chloride (15)

Following the procedure given for the synthesis of $\mathbf{1 1}$ from 1 (200 mg, $0.49 \mathrm{mmol})$ and 14 (333 mg, $0.59 \mathrm{mmol})$ compound 15 (200 mg, 50\%) was obtained as a dark green solid; $R_{F}\left(\mathrm{CHCl}_{3} / \mathrm{MeOH}, 9: 1\right)=0.35 ;$ m.p. $214-218^{\circ} \mathrm{C} ;{ }^{1} \mathrm{H}$ NMR (400 MHz, $\mathrm{CDCl}_{3}$ ): $\delta=8.00$ (s, 1H, 37-H), 7.70 (d, $J$ $=3.8 \mathrm{~Hz}, 1 \mathrm{H}, 39-\mathrm{H}), 7.62(\mathrm{t}, J=3.7 \mathrm{~Hz}, 1 \mathrm{H}, 38-\mathrm{H})$, 7.447.28 (m, 5H, 2'-H, 7'-H, 40-H), 7.01 (d, J=5.4 Hz, 4H, 3'$\left.\mathrm{H}, 8^{\prime}-\mathrm{H}\right), 5.23(\mathrm{t}, J=3.7 \mathrm{~Hz}, 1 \mathrm{H}, 12-\mathrm{H}), 4.47(\mathrm{t}, J=7.9 \mathrm{~Hz}$, $1 \mathrm{H}, 3-\mathrm{H}), 3.70(\mathrm{~m}, 4 \mathrm{H}, 33-\mathrm{H}), 3.38$ (s, 12H, $\left.\mathrm{NMe}_{2}\right), 3.14$ (m, 4H, 34-H), $2.94(\mathrm{~m}, 1 \mathrm{H}, 18-\mathrm{H}), 2.12\left(\mathrm{~m}, 1 \mathrm{H}, 16-\mathrm{H}_{\mathrm{a}}\right)$, 2.02 (s, 3H, 32-H), 1.97-1.79 (m, 2H, 11-H), 1.78-1.45 (m, 16H, 19-H, 6-H, 16-H , 2-H, 15-H, 7-H, 22-H, 21-H, 1-H), 1.44-1.28 (m, 1H, 20-H); 1.19 (m, 2H, 5-H, 9-H), 1.11 (s, $3 \mathrm{H}, 23-\mathrm{H}), 0.89$ (s, 12H, 27-H, 25-H, 24-H), 0.84 (s, 6H, 26-H, 30-H), 0.69 (s, 3H, 29-H) ppm; ${ }^{13} \mathrm{C} \mathrm{NMR} \mathrm{(100} \mathrm{MHz,}$ $\left.\mathrm{CDCl}_{3}\right): \delta=175.1$ (C-28), 171.0 (C-31), 169.1 (C-35), $157.0\left(\mathrm{C}-1^{\prime}, \mathrm{C}-9^{\prime}\right), 144.6$ (C-13), 144.5 (C-36), 140.7 (C-2', $\mathrm{C}^{\prime}$ ), $140.0\left(\mathrm{C}-5^{\prime}\right), 135.8(\mathrm{C}-41), 132.8$ (C-40), $131.2(\mathrm{C}-$ 39), 129.0 (C-38), 127.3 (C-4', C6'), 121.6 (C-12), 114.2 $\left(\mathrm{C}-3^{\prime}, \mathrm{C}^{\prime}\right), 80.9$ (C-3), 55.3 (C-5), 47.7 (C-9), 47.6 (C-17), 47.6 (C-34), 46.3 (C-33), 43.7 (C-18), 43.6 (C-14), 41.9 (C19), 41.3 (C-10), 39.3 (C-8), 39.1 (C-22), 38.2 (C-1), 38.1 (C-7), 37.8 (C-4), 37.7 (C-21), 36.9 (C-15), 33.0 (C-20), 30.3 (C-16), 28.0 (C-11), 27.9 (C-27), 24.0 (C-2), 23.5 (C30), 23.4 (C-32), 21.3 (C-6), 18.2 (C-29), 16.9 (26C), 16.7 (C-23), 15.4 (C-25) ppm; IR (KBr): $\nu=3447 s_{b r}, 2990 w$, $1636 w, 1584 w, 1371 w, 1169 w \mathrm{~cm}^{-1}$; UV-vis $\left(\mathrm{CHCl}_{3}\right): \lambda_{\max }$ $(\log \varepsilon)=220.98 \quad$ (3.54), $346.19 \quad$ (3.87), $484.15 \quad$ (3.76), 682.69 (4.11), 746.26 (4.32) nm; MS (ESI, MeOH): $\mathrm{m} / \mathrm{z}$ $(\%)=921.73\left(100 \%,[\mathrm{M}-\mathrm{Cl}]^{-}\right) ; \mathrm{C}_{26} \mathrm{H}_{81} \mathrm{~N}_{4} \mathrm{O}_{4} \mathrm{Cl}$ (957.78): C 75.24, H 8.52, N 5.85; found: C 75.11, H 8.69, N 5.49.

\section{$N$-(4-((4-dimethylamino)phenyl)-4-((4-(3ß-acetyloxyurs-12- en-28-carboxy)piperazin)-1-yl)oxy)- \\ carbonylphenylmethylencyclohexa-2,5-dien-1-yliden)- $N$ - methylmethanaminium chloride (16)}

Following the procedure given for the synthesis of 11, from 2 (200 mg, $0.49 \mathrm{mmol})$ and $14(333 \mathrm{mg}, 0.59 \mathrm{mmol}) \mathbf{1 6}$ (164 mg, 41\%) was obtained as a green solid; $R_{F}\left(\mathrm{CHCl}_{3} /\right.$ $\mathrm{MeOH}, 9: 1)=0.35$; m.p. $212-215^{\circ} \mathrm{C} ;{ }^{1} \mathrm{H}$ NMR $(400 \mathrm{MHz}$, $\left.\mathrm{CDCl}_{3}\right): \delta=8.01(\mathrm{~d}, J=7.3 \mathrm{~Hz}, 2 \mathrm{H}, 37-\mathrm{H}), 7.56(\mathrm{~d}, J=$ $7.7 \mathrm{~Hz}, 2 \mathrm{H}, 38-\mathrm{H}), 7.38$ (d, $\left.J=7.7 \mathrm{~Hz}, 2 \mathrm{H}, 3^{\prime}-\mathrm{H}\right), 7.08$ (d, $J$ $\left.=9.2 \mathrm{~Hz}, 2 \mathrm{H}, 2^{\prime}-\mathrm{H}\right), 6.99\left(\mathrm{~d}, J=8.2 \mathrm{~Hz}, 2 \mathrm{H}, 7^{\prime}-\mathrm{H}\right), 6.61(\mathrm{~d}$, $\left.J=9.2 \mathrm{~Hz}, 2 \mathrm{H}, 8^{\prime}-\mathrm{H}\right), 5.25(\mathrm{t}, J=3.5 \mathrm{~Hz}, 3 \mathrm{H}, 12-\mathrm{H}), 4.46$ (t, $J=8.0 \mathrm{~Hz}, 1 \mathrm{H}, 3-\mathrm{H}), 3.74(\mathrm{~m}, 4 \mathrm{H}, 33-\mathrm{H}), 3.38(\mathrm{~s}, 6 \mathrm{H}$, $\left.1^{\prime}-\mathrm{NMe}_{2}\right), 3.10(\mathrm{q}, 7.5 \mathrm{~Hz}, 5 \mathrm{H}, 34-\mathrm{H}, 18-\mathrm{H}), 2.15$ (s, 1H, $\left.16-\mathrm{H}_{\mathrm{a}}\right), 2.02(\mathrm{~s}, 3 \mathrm{H}, 32-\mathrm{H}), 1.96-1.46\left(\mathrm{~m}, 4 \mathrm{H}, 11-\mathrm{H}, 6-\mathrm{H}_{\mathrm{a}}\right.$, $\left.16-\mathrm{H}_{\mathrm{b}}\right), 1.39\left(\mathrm{~m}, 8 \mathrm{H}, 2-\mathrm{H}, 15-\mathrm{H}_{\mathrm{a}}, 19-\mathrm{H}, 7-\mathrm{H}, 9-\mathrm{H}, 21-\mathrm{H}_{\mathrm{a}}\right)$, $1.28-1.14\left(\mathrm{~m}, 9 \mathrm{H}, 20-\mathrm{H}, 6-\mathrm{H}_{\mathrm{b}}, 22-\mathrm{H}, 21-\mathrm{H}_{\mathrm{b}}, 1-\mathrm{H}, 15-\mathrm{H}_{\mathrm{b}}, 5-\right.$ H), 1.12 (s, 3H, 27-H), 0.90 (s, 3H, 30-H), 0.89 (s, 3H, 25H), 0.85 (s, 3H, 29-H), 0.83 (s, 3H, 24-H), 0.71 (s, 3H, 23$\mathrm{H}), 0.65$ (s, 3H, 26-H) ppm; ${ }^{13} \mathrm{C} \mathrm{NMR}\left(100 \mathrm{MHz}, \mathrm{CDCl}_{3}\right)$ : $\delta=175.3(\mathrm{C}-28), 171.0(\mathrm{C}-31), 169.2(\mathrm{C}-35), 157.0\left(\mathrm{C}-1^{\prime}\right.$, C-9'), $144.7(\mathrm{C}-13), 144.5\left(\mathrm{C}-7^{\prime}\right), 140.9\left(\mathrm{C}-8^{\prime}\right), 140.8(\mathrm{C}-$ 2'), 139.4 (C-36), 134.7 (C-37), 127.2 (C-38), 121.6 (C-12), $114.1\left(\mathrm{C}-3^{\prime}\right), 80.9$ (C-3), 55.3 (C-30), 52.5 (C-18), 47.7 (C17), 47.6 (C-9), 47.6 (C-34), $45.9\left(\mathrm{NMe}_{2}\right), 43.6$ (C-33), 43.5 (C-14), 41.9 (C-8), $41.3\left(9^{\prime}-\mathrm{NMe}_{2}\right), 39.1$ (C-5), 38.1 (C-20), 37.6 (C-1), 36.9 (C-4), 33.9 (C-10), 33.0 (C-22), 30.4 (C-21), 29.9 (C-15), 28.0 (C-24), 27.8 (C-16), 25.9 (C11), 24.1 (C-27), 23.5 (C-2), 23.4 (C-19), 21.3 (C-32), 18.2 (C-29), 16.9 (C-23), 16.6 (C-26), 15.4 (C-25), 8.7 (C-7), 0.9 $\left(\mathrm{C}-5^{\prime}\right) \mathrm{nm}$; IR (KBr): $\nu=3442 s_{b r}, 2940 m, 2678 m, 1618 m$, $1584 m, 1371 \mathrm{~m}, 1248 w, 1171 \mathrm{~m} \mathrm{~cm}^{-1}$; UV-vis $\left(\mathrm{CHCl}_{3}\right)$ : $\lambda_{\max }(\log \varepsilon)=226.9$ (3.9), 256.0 (4.1), 486.2 (3.98), 682 (4.21), 737.0 (4.6) nm; MS (ESI, MeOH): $m / z(\%)=921.73$ $\left(100 \%,[\mathrm{M}-\mathrm{Cl}]^{-}\right) ; \mathrm{C}_{26} \mathrm{H}_{81} \mathrm{~N}_{4} \mathrm{O}_{4} \mathrm{Cl}$ (957.78): $\mathrm{C} 75.24, \mathrm{H}$ 8.52, N 5.85; found: C 74.99, H 8.68, N 5.44.

Acknowledgements We like to thank Dr D. Ströhl and his team for the NMR spectra and the late Dr R. Kluge for numerous ESI-MS measurements. IR and UV-vis spectra were recorded by V. Simon. The cell lines were provided by Dr T. Müller. Open Access funding provided by Projekt DEAL.

\section{Compliance with ethical standards}

Conflict of interest The authors declare that they have no conflict of interest.

Publisher's note Springer Nature remains neutral with regard to jurisdictional claims in published maps and institutional affiliations.

Open Access This article is licensed under a Creative Commons Attribution 4.0 International License, which permits use, sharing, adaptation, distribution and reproduction in any medium or format, as long as you give appropriate credit to the original author(s) and the source, provide a link to the Creative Commons license, and indicate if changes were made. The images or other third party material in this article are included in the article's Creative Commons license, unless indicated otherwise in a credit line to the material. If material is not included in the article's Creative Commons license and your intended use is not permitted by statutory regulation or exceeds the permitted use, you will need to obtain permission directly from the copyright holder. To view a copy of this license, visit http://creativecommons. org/licenses/by/4.0/.

\section{References}

Biedermann D, Eigenrova B, Hajduch M, Sarek J (2010) Synthesis and evaluation of biological activity of the quaternary ammonium 
salts of lupane-, oleanane-, and ursane-type acids. Synthesis 3839-3848

Brandes B, Hoenke S, Fischer L, Csuk R (2020) Design, synthesis and cytotoxicity of BODIPY FL labelled triterpenoids. Eur J Med Chem 185:111858

Cai H, Wahajuddin M, Everett R, Thakker DR (2014) Do cationselective transporters help or hurt the antitumor efficacy of metformin in breast cancer. Cancer Res 74:4639

Cai H, Zhang YH, Han TX, Everett RS, Thakker DR (2016) Cationselective transporters are critical to the AMPK-mediated antiproliferative effects of metformin in human breast cancer cells. Intern J Cancer 138:2281-2292

Costero AM, Parra M, Gil S, Gotor R, Martinez-Manez R, Sancenon F, Royo S (2012) Selective detection of nerve agent simulants by using triarylmethanol-based chromogenic chemodosimeters. Eur J Org Chem 26:4937-4946

Everett RS, Zhang YH, Ononiwu IM, Bae-Jump VL, Thakker DR (2013) Multiple cation-selective transporters contribute to the antiproliferative effects of metformin in ovarian cancer cell lines. Cancer Res 73. https://doi.org/10.1158/1538-7445.AM2013-4427

Harle JP, Arata S, Mine S, Kamegawa S, Nguyen VT, Maeda T, Nakazumi H, Fujiwara H (2018) Malachite green derivatives for dye-sensitized solar cells: optoelectronic characterizations and persistence on $\mathrm{TiO}_{2}$. Bull Chem Soc Jpn 91:52-64

Holzel D, Eckel R, Bauerfeind I, Baier B, Beck T, Braun M, Ettl J, Hamann U, Kiechle M, Mahner S, Schindlbeck C, de Waal J, Harbeck N, Engel J (2017) Improved systemic treatment for early breast cancer improves cure rates, modifies metastatic pattern and shortens post-metastatic survival: 35-year results from the Munich Cancer Registry. J Cancer Res Clin Oncol 143:1701-1712

Kahnt M, Wiemann J, Fischer L, Sommerwerk S, Csuk R (2018) Transformation of asiatic acid into a mitocanic, bimodal-acting rhodamine B conjugate of nanomolar cytotoxicity. Eur J Med Chem 159:143-148

Kataev VE, Strobykina IY, Zakharova LY (2014) Quaternary ammonium derivatives of natural terpenoids. Synthesis and properties. Russ Chem Bull 63:1884-1900

Krajcovicova S, Stankova J, Dzubak P, Hajduch M, Soural M, Urban M (2018) A synthetic approach for the rapid preparation of BODIPY conjugates and their use in imaging of cellular drug uptake and distribution. Chem Eur J 24:4957-4966

Laffman-Johnson E (2012) Improving survival and cure rates of breast cancer patients. Clin Pharm Ther 92:272-272

Mueller W, Hattesohl I, Schuetz HJ, Meyer G (1981) Polyethylene glycol derivatives of base and sequence specific DNA ligands: DNA interaction and application for base specific separation of DNA fragments by gel electrophoresis. Nucl Acids Res 9:95-119

Müller M, Kruse L, Tabrett AM, Barbara DJ (1997) Detection of a single base exchange in PCR-amplified DNA fragments using agarose gel electrophoresis containing bisbenzimide-PEG. Nucl Acids Res 25:5125-5126

Qian CY, Zheng Y, Wang Y, Chen J, Liu JY, Zhou HH, Yin JY, Liu ZQ (2016) Associations of genetic polymorphisms of the transporters organic cation transporter 2 (OCT2), multidrug and toxin extrusion 1 (MATE1), and ATP-binding cassette subfamily C member 2 (ABCC2) with platinum-based chemotherapy response and toxicity in non-small cell lung cancer patients. Chin J Cancer 35. https://doi.org/10.1186/s40880-016-0145-8

Rassow B, Gruber H (1915) p-cyano- und p-carboxymalachitgrün. J Prakt Chem 91:341-357

Sinev VV, Proskuryakova TV, Aleksandr AV, Ginzburg OF (1978) Study of arylmethane dyes. XIX. Study of the effect of ionic strength on the kinetics of triarylcarbinol formation. Zh Org Khim 14:1676-1681

Sommerwerk S, Heller L, Kerzig C, Kramell AE, Csuk R (2017) Rhodamine $\mathrm{B}$ conjugates of triterpenoic acids are cytotoxic mitocans even at nanomolar concentrations. Eur J Med Chem 127:1-9

Spivak AY, Nedopekina DA, Khalitova RR, Gubaidullin RR, Odinokov VN, Bel'skii YP, Bel'skaya NV, Khazanov VA (2017) Triphenylphosphonium cations of betulinic acid derivatives: synthesis and antitumor activity. Med Chem Res 26:518-531

Spivak AY, Nedopekina DA, Shakurova ER, Khalitova RR, Gubaidullin RR, Odinokov VN, Dzhemilev UM, Bel'skii YP, Bel'skaya NV, Stankevich SA, Korotkaya EV, Khazanov VA (2013) Synthesis of lupane triterpenoids with triphenylphosphonium substituents and studies of their antitumor activity. Russ Chem Bull 62:188-198

Vliek S, Jager A, Jonge-Lavrencic M, Lotz JP, Goncalves A, Graeser M, Nitz U, Mandjes IAM, Holtkamp MJ, Schot M, Retel VP, Kuip EJ, Wymenga MN, Konings IR, Tjan-Heijnen VCG, Kroep JR, Schröder CP, Van der Wall E, Linn SC (2018) Substantially improving the cure rate of high-risk BRCA1-like breast cancer patients with personalized therapy (SUBITO) - an international randomized phase III trial. Cancer Res 78. https://doi.org/10. 1158/1538-7445.SABCS17-OT2-07-08

Wolfram RK, Fischer L, Kluge R, Ströhl D, Al-Harrasi A, Csuk R (2018a) Homopiperazine-rhodamine B adducts of triterpenoic acids are strong mitocans. Eur J Med Chem 155:869-879

Wolfram RK, Heller L, Csuk R (2018b) Targeting mitochondria: esters of rhodamine $\mathrm{B}$ with triterpenoids are mitocanic triggers of apoptosis. Eur J Med Chem 152:21-30

Yang MC, Fang JM, Kuo TF, Wang DM, Huang YL, Liu LY, Chen $\mathrm{PH}$, Chang TH (2007) Production of antibodies for selective detection of malachite green and the related triphenylmethane dyes in fish and fishpond water. J Agric Food Chem 55:8851-8856

Zhao C, Fernandez A, Avlonitis N, Vande Velde G, Bradley M, Read ND, Vendrell M (2016) Searching for the optimal fluorophore to label antimicrobial peptides. ACS Comb Sci 18:689-696 